\title{
Interpretation of the space-bandwidth product as the entropy of distinct connection patterns in multifacet optical interconnection architectures
}

\author{
Haldun M. Ozaktas \\ Department of Electrical Engineering, Bilkent University, 06533 Bilkent, Ankara, Turkey \\ K.-H. Brenner and Adolf W. Lohmann \\ Lehrstuhl Angewandte Optik, Physics Institute, University of Erlangen-Nürnberg, Staudstrasse 7/B2, \\ W-8520 Erlangen, Germany
}

Received July 22, 1992; revised manuscript received October 13, 1992; accepted October 16, 1992

We show that the entropy of the distinct connection patterns that are possible with multifacet optical interconnection architectures is approximately equal to the space-bandwidth product of the optical system.

\section{INTRODUCTION}

The importance of the number of distinct connection patterns that can be wired up between $N$ input and $N$ output nodes was emphasized by Keyes. ${ }^{1,2}$ In the context of microelectronic packaging, the input and output nodes may be the terminals of logic gates on a chip or the pinouts of chips on a multichip carrier. In the context of an optical interconnection system, the input nodes may be optical sources and the output nodes optical detectors on the surface of electronic processing elements. The number $Q$ of different ways in which the input nodes can be connected to the output nodes is a measure of the flexibility afforded by that interconnection system. A designer confronted with the task of implementing a particular connection pattern or circuit diagram has a much greater chance of success if $Q$ is larger. Because $Q$ can be extremely large, it is more convenient to work with its logarithm, $\Omega=$ $\log _{2} Q$, which can be interpreted as the information (entropy) required to specify a particular connection pattern out of all the possible patterns. For instance, for a full crossbar interconnection network, where each input can be tied to any set of outputs,

$$
\begin{aligned}
& Q=\left[\sum_{n=0}^{N} C(N, n)\right]^{N}=\left(2^{N}\right)^{N}=2^{N^{2}}, \\
& \Omega=N^{2},
\end{aligned}
$$

since each of the $N$ inputs has the choice of being connected to $0 \leq n \leq N$ output nodes in $C(N, n)$ distinct ways $\{C(x, y) \equiv x ! /[y !(x-y) !]\}$. An alternative way of looking at this is that there is a binary decision (to connect or not to connect) between each of the $N^{2}$ possible input-output pairs.

Here we discuss the number of distinct connection patterns that are possible with a quite general class of freespace optical interconnection architectures, discussed previously by several authors. ${ }^{3-13}$ The generic form of the architecture is depicted in Fig. 1, several other essentially equivalent forms being possible. The light originating from a particular input channel is made to fall on any number of filter facets on the filter (Fourier) plane, through the use of appropriate phase elements corresponding to the desired positional shift in the Fourier plane. (Figure 1 shows the use of prisms as phase elements; if the light is intended to fall on more than one filter facet, multiple gratings should be used.) Each filter facet is assumed to be a phase element corresponding to a single distinct displacement between the input and output planes. All input channels using the same filter facet will be displaced by the same relative amount in the output plane. If one's goal is to displace two input channels by different magnitudes or directions in the output plane, different filter facets must be used. Hence this architecture allows partially space-variant mapping of the inputs to the outputs. ${ }^{13}$

The main result of the research presented in this paper is that the value of $\Omega$ for this architecture is approximately equal to the space-bandwidth product of the optical system. This result provides a new interpretation of the space-bandwidth product, which is a measure of the throughput and the cost of the system. In Section 2 we derive this result in a simple manner by ignoring edge effects. In Section 3 we provide a more complicated derivation that takes edge effects into account, which results in a slightly different but essentially identical result. Most readers will prefer to disregard this section, which has been included for mathematical completeness.

\section{ANALYSIS}

We assume that each of the $M$ filter facets corresponds to a distinct relative displacement between the input and output planes and that at least one input channel employs each filter facet. (If these conditions are not satisfied, the number of filter facets could be reduced without decreasing the number of distinct connection patterns that 

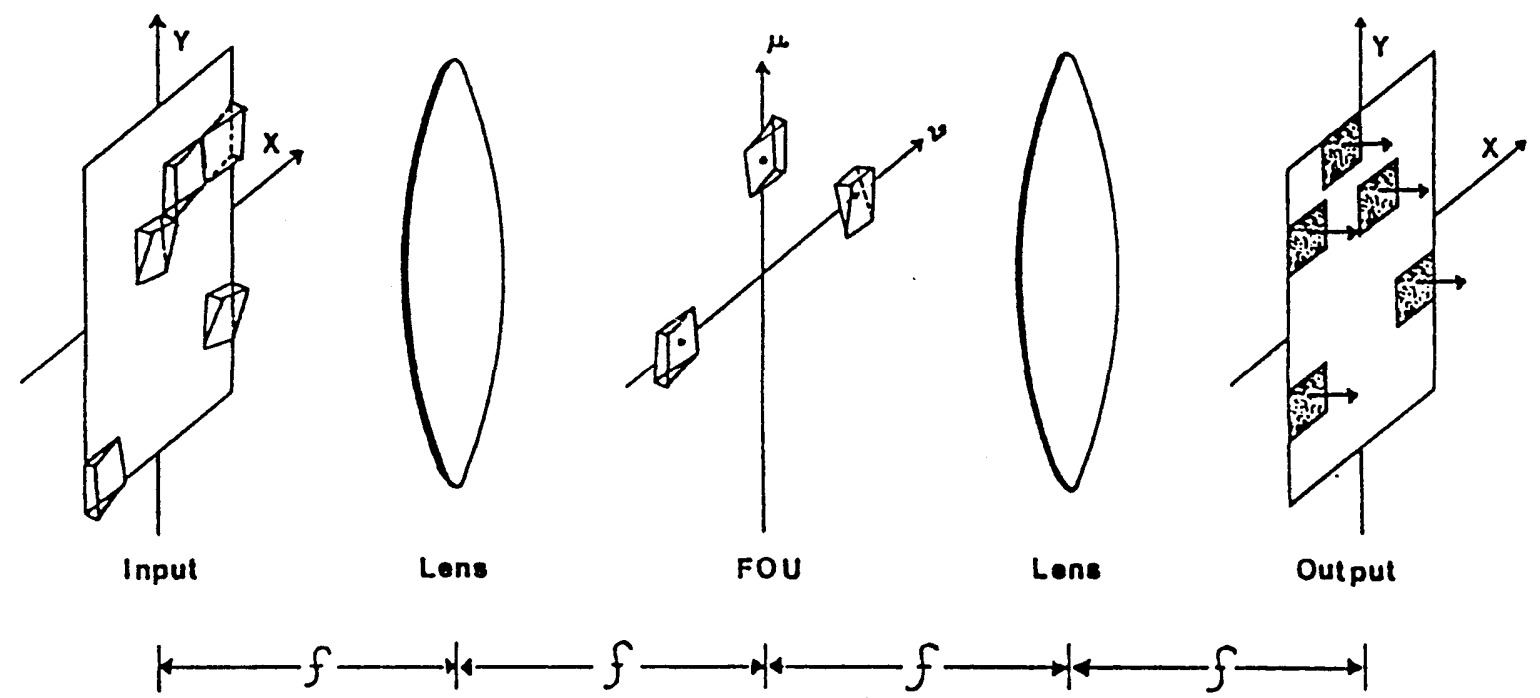

Fig. 1. Multifacet, partially space-variant optical interconnection architecture. $f$ is the focal length of the lenses. There are $N$ channels in the input and output planes, and there are $M$ filter facets. For simplicity we assume that these are arrayed in the Cartesian manner.

are possible. The use of more than one filter facet with the same displacement, or leaving a filter facet unused, is simply wasteful.) Since there are $N$ output channels, at most $N$ distinct displacements are possible for each input (directed toward each of the $N$ outputs), so that if each filter facet is to be distinct, $M \leq N$.

[In fact, the set of possible displacements for a particular input channel depends on its location in the input plane. Although $N$ distinct displacements are possible for each input (directed toward each of the $N$ outputs), a total of $\simeq 4 N$ distinct displacements is possible when we consider all input channels. In the present derivation, we are assuming that all input and output channels can be treated uniformly regardless of their location in the input or output plane. A more exact analysis is presented in Section 3.]

For simplicity, the area and shape of the numerous input and output channels and those of the numerous filter facets are assumed to be identical (as in Fig. 1). Let the area of each input channel be denoted by $a$ so that the area of the input plane is $\mathrm{Na}$ (in square meters), and let the area of each filter facet be denoted by $\alpha$ so that the area of the filter plane is $M \alpha$ (in reciprocal square meters). The space-bandwidth product of the system $(S B)$ is equal to the product of the area of the input plane and the area of the filter plane, $S B=N M a \alpha$. Also, the area of the input channels and the filter facets must satisfy $a \alpha \geq \kappa$, where $\kappa$ is a coefficient of the order of unity, because the light distribution at the input channel and that at the filter facet form a Fourier-transform pair. Thus we have ${ }^{13}$

$$
N M \leq S B^{\prime} \equiv S B / \kappa .
$$

We assume that the space-bandwidth product of the system is not underutilized, so that the above relation is satisfied with equality. Since $M \leq N$, it also follows that $M \leq \sqrt{S B^{\prime}}$ and $N \geq \sqrt{S B^{\prime}}$.

We allow for arbitrary fan-out at the input plane and arbitrary fan-in at the output plane as well as the possibility of some nodes not being involved in any connections.
(As mentioned above, for fan-out to be established, the prisms in Fig. 1 must be replaced by multiple gratings.)

Between 1 and $N$ input channels may utilize the same filter facet. All these channels will receive the same displacement in the output plane. $i$ out of $N$ channels may utilize the same displacement in $C(N, i)$ distinct ways. Summing over $i$, we obtain

$$
\sum_{i=1}^{N} C(N, i)=2^{N}-1
$$

Since the same multiplicity exists for each filter facet, there are $\left(2^{N}-1\right)^{M} \simeq 2^{N M}$ distinct ways in which the $N$ input channels can be mapped to the $M$ displacements.

(In fact, all the $N$ input channels cannot utilize a given displacement. For instance, a channel at the rightmost edge of the input plane cannot utilize displacement vectors with positive components in the $x$ direction because this would result in its being imaged outside the output plane. The greater the magnitude of the displacement, the smaller the number of input channels that can utilize it. As is mentioned above, we are ignoring such edge effects in the present derivation.)

Now we must determine the number of ways in which $M$ distinct displacements can be chosen out of $N$ possible displacements. This is simply $C(N, M)$ since the particular order in which the displacements are assigned to the filter facets is not important. [If the displacements associated with two filter facets are interchanged, this can be compensated by adjusting the deflection components at the input plane that select the filter facet(s) that each input channel will use. Thus the two situations would not correspond to distinct connection patterns.]

Therefore the number of distinct connection patterns that can be implemented with $M$ filter facets is given by

$$
\begin{aligned}
& Q=C(N, M) 2^{N M}, \\
& \Omega=\log _{2} C(N, M)+N M=\log _{2} C(N, M)+S B^{\prime} .
\end{aligned}
$$

The function $C(N, M)$ will be approximately at its maximum value when $M \simeq N / 2$, rounded to the nearest in- 
teger. Using $N M=S B^{\prime}$, we find that $M \simeq \sqrt{S B^{\prime} / 2}$ and $N \simeq \sqrt{2 S B^{\prime}}$. Since

$$
\sum_{M=0}^{N} C(N, M)=2^{N}
$$

and thus

$$
\log _{2} \sum_{M=0}^{N} C(N, M)=N,
$$

we have $\log _{2} \mathrm{C}(N, M) \leq N \simeq \sqrt{2 S B^{\prime}}$, which is $\ll S B^{\prime}$ because $S B^{\prime} \gg 1$. Since even the maximum value of $\log _{2} C(N, M) \ll S B^{\prime}$, we obtain

$$
\Omega \simeq S B^{\prime},
$$

which is our main result. In the simplest possible terms this can be understood by noting that there is a binary choice involved in whether we connect input channel $i$ to filter facet $j$, and since $1 \leq i \leq N$ and $1 \leq j \leq M$, there are a total of $N M=S B^{\prime}$ binary decisions, which is nothing but the definition of $\Omega$. To arrive at relation (4), it was only necessary to show that the additional multiplicity contributed by the number of distinct ways in which a subset of the possible displacements are assigned to the $M$ filter facets is insignificant.

It is also interesting to investigate what fraction $\eta$ of the total number of possible connection patterns $2^{N^{2}}$ (which can all be realized with a full crossbar architecture) we are able to realize with this architecture. Dividing $Q$ by $2^{N^{2}}$, we obtain

$$
\eta=\frac{2^{N M} C(N, M)}{2^{N^{2}}} \leq 1,
$$

which is satisfied with equality when $M=N$. Taking logarithms and remembering that the term involving $C(N, M)$ is negligible,

$$
\log _{2} \eta=N(M-N)=S B^{\prime}-N^{2} \leq 0,
$$

which is satisfied with equality when $M=N$. For a given space-bandwidth product, the number of distinct connection patterns that are possible with our architecture is fixed and cannot be increased by increasing either $N$ or $M$. By increasing $N$, the number of potential connection patterns $2^{N^{2}}$ can be increased, but since the number of connection patterns that can be implemented with our architecture is fixed, the ratio $\eta$ will be decreased.

Since we assume that each filter facet corresponds to a distinct displacement and is utilized for at least one connection, $Q(M)$ is the number of distinct connection patterns that can be implemented with exactly $M$ filter facets; the connection patterns that can be implemented with less than $M$ filter facets are not included. For a given value of $N$, the space-bandwidth product determines the maximum number of filter facets $M$ that we can afford to have $\left(S B^{\prime} / N\right)$. The total number of distinct connection patterns that can be implemented with at most $M=$ $S B^{\prime} / N$ filter facets is

$$
\sum_{i=1}^{M} 2^{N i} \simeq 2^{N M}=2^{S D^{\prime}} .
$$

The geometric series is dominated by and almost equal to its largest term. This means that almost all the connec- tion patterns that can be implemented with at most $M$ filter facets require exactly $M$ filter facets. A corollary is that almost all the $2^{N^{2}}$ connection patterns that are possible with a full crossbar require that $M=N$ facets be used to be implemented with the architecture under consideration. The number of connection patterns for which a smaller number of facets are sufficient is negligible in comparison.

\section{CONSIDERATION OF EDGE EFFECTS}

In this section we repeat the above derivation, taking into account edge effects.

Remember that we assume that the input and output planes consist of Cartesian arrays of $\sqrt{N} \times \sqrt{N}$ channels. As discussed above, there are at most $N$ distinct displacements that are possible for each input. However, when all input channels are considered, the total number of distinct displacement vectors possible is $(2 \sqrt{N}-1)^{2} \simeq 4 N$. (This is the number of distinct vectors that can be drawn between pairs of points in a $\sqrt{N} \times \sqrt{N}$ array.) A given input cannot utilize $\simeq 3 N$ out of these $\simeq 4 N$ displacements since they would image the input outside of the array of $\sqrt{N} \times \sqrt{N}$ output channels.

First we establish an upper bound for $\Omega$. We can choose $M$ displacements out of the $\approx 4 N$ possible in $C(4 N, M)$ distinct ways. In Section 2 we calculated that the input channels can be mapped to a particular set of $M$ displacements in $2^{N M}$ ways, assuming that any number of input channels between 1 and $N$ could utilize each displacement. However, since in fact all $N$ input channels cannot utilize a given displacement, this was an overestimate. Thus we are led to the upper bound

$\Omega \leq \log _{2} C(4 N, M)+N M=\log _{2} C(4 N, M)+S B^{\prime} \simeq S B^{\prime}$,

where in the last step a similar argument as used in arriving at relation (4) is invoked.

Now we derive a lower bound for $\Omega$. Let $(i, j)$ denote the horizontal and vertical components of a particular displacement vector, which must satisfy

$$
\begin{aligned}
& -(\sqrt{N}-1) \leq i \leq(\sqrt{N}-1), \\
& -(\sqrt{N}-1) \leq j \leq(\sqrt{N}-1) .
\end{aligned}
$$

Without loss of generality, assume for the moment that $i, j \geq 0$. Let $(k, l)$ denote the coordinates of a particular input channel such that $1 \leq k \leq \sqrt{N}$ and $1 \leq l \leq \sqrt{N}$. If the values of $k$ or $l$ are large, this input may not be able to utilize the given displacement because doing so would cause the displacement to be imaged outside the array of output channels. More precisely, only those inputs satisfying

$$
\begin{aligned}
& 1 \leq k \leq \sqrt{N}-i, \\
& 1 \leq l \leq \sqrt{N}-j
\end{aligned}
$$

can utilize the displacement with components $(i, j)$. The maximum number of inputs that can utilize this particular displacement is $(\sqrt{N}-|i|)(\sqrt{N}-|j|)$, which is exactly the number of inputs contained in the rectangle bound by relations (11) and (12), and the absolute values will also 
ensure validity for negative values of $i$ and $j$. Any number of inputs between 1 and $(\sqrt{N}-|i|)(\sqrt{N}-|j|)$ may utilize this particular displacement, in $\left[2^{(\sqrt{N}-|i|)(\sqrt{N}-|j|)}-1\right]$ distinct ways.

Now assume that we have chosen a particular set of $M$ distinct displacements out of the $\simeq 4 N$ possible displacements. The total number of distinct ways in which the inputs can be mapped to these $M$ displacements is then

$$
\prod\left[2^{(\sqrt{N}-|i|)(\sqrt{N}-|j|)}-1\right]
$$

where the product is taken over all the $M$ displacements. (This step corresponds to taking the $M$ th power of $2^{N}-1$ in Section 2.) The total number of distinct connection patterns is then

$$
Q=\sum \prod\left[2^{(\sqrt{N}-|i|)(\sqrt{N}-|j|)}-1\right]
$$

where the sum is over the $C(4 N, M)$ different possible sets of $M$ displacements. [This step corresponds to multiplying by $C(N, M)$ in Section 2 .]

Because the last expression is difficult to evaluate, we must satisfy ourselves with a lower bound. Any term in the above summation is a lower bound for $Q$; hence we choose a term that is both easy to evaluate and as large as possible, one that corresponds to a set of displacements that are symmetric and as short as possible \{since a greater number of inputs can utilize shorter displacements [relations (11) and (12)]\}. More specifically, we choose the set of $M$ displacement vectors with components $(i, j)$, satisfying

$$
\begin{aligned}
& -(\sqrt{M}-1) / 2 \leq i \leq(\sqrt{M}-1) / 2, \\
& -(\sqrt{M}-1) / 2 \leq j \leq(\sqrt{M}-1) / 2 .
\end{aligned}
$$

Thus we can now explicitly express the product and write a lower bound for $Q$,

$$
Q \geq \prod_{j} \prod_{i}\left[2^{(\sqrt{N}-|i|)(\sqrt{N}-|j|)}-1\right]
$$

where the ranges of the products are as given by relations (15) and (16). Taking logarithms,

$$
\Omega \geq \sum_{j} \sum_{i} \log _{2}\left[2^{(\sqrt{N}-|i|)(\sqrt{N}-|j|)}-1\right]
$$

If we ignore the -1 in the square brackets, relation (18) is clearly an excellent approximation unless $|i|$ and $|j|$ are close to $\sqrt{N}$. $\quad|i|$ and $|j|$ will attain their maximum values $\sqrt{N}-1$ only when $M$ attains its maximum value $(2 \sqrt{N}-1)^{2} \simeq 4 N$. Even in this case the overall error incurred by ignoring the -1 in the square brackets will be quite small because the terms that are most affected are few in number and small in magnitude compared with the terms for which ignoring the -1 is an excellent approximation, which are many in number and large in magnitude. Thus

$$
\Omega \geq \sum_{j} \sum_{i}(\sqrt{N}-|i|)(\sqrt{N}-|j|)
$$

The right-hand side can be calculated as

$$
\begin{aligned}
{\left[\sum_{i}(\sqrt{N}-|i|)\right]^{2} } & =\left[\sqrt{M N}-2 \sum_{i=1}^{(\sqrt{M}-1) / 2} i\right]^{2} \\
& =\left(\sqrt{M N}-\frac{M-1}{4}\right)^{2} .
\end{aligned}
$$

Thus, using $M N=S B^{\prime}$, we obtain

$$
\Omega \geq\left(\sqrt{S B^{\prime}}-M / 4+1 / 4\right)^{2} .
$$

Since $M \leq(2 \sqrt{N}-1)^{2}<4 N$, we have $N>M / 4$; hence $S B^{\prime}=M N>M^{2} / 4$ and $M<2 \sqrt{S B^{\prime}}$. Noting that the right-hand side of relation (21) is a decreasing function of $M$,

$$
\begin{aligned}
\left(\sqrt{S B^{\prime}}-M / 4+1 / 4\right)^{2} & >\left(\sqrt{S B^{\prime}}-2 \sqrt{S B^{\prime}} / 4+1 / 4\right)^{2} \\
& >S B^{\prime} / 4,
\end{aligned}
$$

so that

$$
\Omega>\frac{S B^{\prime}}{4}
$$

Combining our lower and upper bounds, we have $S B^{\prime} / 4 \leq \Omega \leq S B^{\prime}$. Hence, apart from a numerical factor of the order of unity, $\Omega \sim S B^{\prime}$.

\section{CONCLUSION}

We have shown that the value of $\Omega$ for the quite general class of multifacet partially space-variant free-space optical interconnection architectures described in this paper is approximately equal to the space-bandwidth product of the optical system. Thus we have provided a new interpretation of the space-bandwidth product.

$\Omega$ is a measure of the flexibility of a particular interconnection architecture in being able to accommodate a given connection pattern or circuit diagram. The spacebandwidth product is a measure of the throughput of an optical system as limited by physical considerations and is also closely related to the cost of the system. By showing that these two quantities are essentially equivalent, we have provided a bridge between physical concepts and architectural-circuit concepts.

Finally we note that, although we have assumed that we are dealing with a fixed connection pattern, the system could be dynamically reconfigured if active deflectors are employed at the inputs and outputs.

\section{ACKNOWLEDGMENT}

H. M. Ozaktas acknowledges the support of the Alexander von Humboldt Foundation through a postdoctoral research fellowship.

\section{REFERENCES}

1. R. W. Keyes, "Communication in computation," Int. J. Theor. Phys. 21, 263-273 (1982).

2. R. W. Keyes, The Physics of VLSI Systems (Addison-Wesley, Reading, Mass., 1987).

3. E. Bradley, P. K. L. Yu, and A. R. Johnston, "System issues relating to laser diode requirements for VLSI holographic optical interconnects," Opt. Eng. 28, 201-211 (1989). 
4. G. E. Lohman and A. W. Lohmann, "Optical interconnection network utilizing diffraction gratings," Opt. Eng. 27, 893-900 (1988).

5. R. K. Kostuk, J. W. Goodman, and L. Hesselink, “Optical imaging applied to microelectronic chip-to-chip interconnections," Appl. Opt. 24, 2851-2858 (1985).

6. R. K. Kostuk, "Multiple grating volume reflection holograms with application to optical interconnects," Ph.D. dissertation (Stanford University, Stanford, Calif., 1986).

7. M. R. Feldman, C. C. Guest, T. J. Drabik, and S. C. Esener, "Comparison between electrical and free space optical interconnects for fine grain processor arrays based on interconnect density capabilities," Appl. Opt. 28, 3820-3829 (1989).

8. M. R. Feldman and C. C. Guest, "Interconnect density capabilities of computer generated holograms for optical interconnection of very large scale integrated circuits," Appl. Opt. 28, 3134-3137 (1989).
9. H. M. Ozaktas and J. W. Goodman, "Lower bound for the communication volume required for an optically interconnected array of points," J. Opt. Soc. Am. A 7, 2100-2106 (1990).

10. H. M. Ozaktas, Y. Amitai, and J. W. Goodman, "Comparison of system size for some optical interconnection architectures and the folded multi-facet architecture," Opt. Commun. 82, 225-228 (1991).

11. H. M. Ozaktas, Y. Amitai, and J. W. Goodman, "A three dimensional optical interconnection architecture with minimal growth rate of system size," Opt. Commun. 85, 1-4 (1991).

12. H. M. Ozaktas, "A physical approach to communication limits in computation," Ph.D. dissertation (Stanford University, Stanford, Calif., 1991).

13. G. E. Lohman and K.-H. Brenner, "Space-variance in optical computing systems," Optik (Stuttgart) 89, 123-134 (1992). 